\title{
Operational utility of the reverse- transcription recombinase polymerase amplification for detection of dengue virus
}

Kim-Kee Tan ${ }^{1,2,3}$, Noor Syahida Azizan ${ }^{1,2}$, Che Norainon Yaacob ${ }^{1,2}$, Nurul Asma Anati Che Mat Seri ${ }^{1,2}$, Nur Izyan Samsudin ${ }^{1,2}$, Boon-Teong Teoh ${ }^{1,2}$, Sing-Sin Sam ${ }^{1,2}$ and Sazaly AbuBakar ${ }^{1,2,3^{*}}$ (i)

\begin{abstract}
Background: A method for rapid detection of dengue virus using the reverse-transcription recombinase polymerase amplification (RT-RPA) was recently developed, evaluated and made ready for deployment. However, reliance solely on the evaluation performed by experienced researchers in a well-structured and well-equipped reference laboratory may overlook the potential intrinsic problems that may arise during deployment of the assay into new application sites, especially for users unfamiliar with the test. Appropriate assessment of this newly developed assay by users who are unfamiliar with the assay is, therefore, vital.
\end{abstract}

Methods: An operational utility test to elucidate the efficiency and effectiveness of the dengue RT-RPA assay was conducted among a group of researchers new to the assay. Nineteen volunteer researchers with different research experience were recruited. The participants performed the RT-RPA assay and interpreted the test results according to the protocol provided. Deviation from the protocol was identified and tabulated by trained facilitators. Post-test questionnaires were conducted to determine the user satisfaction and acceptability of the dengue RT-RPA assay.

Results: All the participants completed the test and successfully interpreted the results according to the provided instructions, regardless of their research experience. Of the 19 participants, three (15.8\%) performed the assay with no deviations and 16 (84.2\%) performed the assay with only 1 to 5 deviations. The number of deviations from protocol, however, was not correlated with the user laboratory experience. The accuracy of the results was also not affected by user laboratory experience. The concordance of the assay results against that of the expected was at $89.3 \%$. The user satisfaction towards the RT-RPA protocol and interpretation of results was $90 \%$ and $100 \%$, respectively.

Conclusions: The dengue RT-RPA assay can be successfully performed by simply following the provided written instructions. Deviations from the written protocols did not adversely affect the outcome of the assay. These suggest that the RT-RPA assay is indeed a simple, robust and efficient laboratory method for detection of dengue virus. Furthermore, high new user acceptance of the RT-RPA assay suggests that this assay could be successfully deployed into new laboratories where RT-RPA was not previously performed.

Keywords: Infectious diseases, Diagnostics, Dengue, Isothermal, PCR, RT-RPA

\footnotetext{
*Correspondence: sazaly@um.edu.my

${ }^{1}$ Tropical Infectious Diseases Research and Education Centre (TIDREC),

University of Malaya, 50603 Kuala Lumpur, Malaysia

${ }^{2}$ WHO Collaborating Centre for Arbovirus Research and Reference (Dengue

and Severe Dengue), University of Malaya, 50603 Kuala Lumpur, Malaysia

Full list of author information is available at the end of the article
}

(c) The Author(s). 2018 Open Access This article is distributed under the terms of the Creative Commons Attribution 4.0 International License (http://creativecommons.org/licenses/by/4.0/), which permits unrestricted use, distribution, and reproduction in any medium, provided you give appropriate credit to the original author(s) and the source, provide a link to the Creative Commons license, and indicate if changes were made. The Creative Commons Public Domain Dedication waiver (http://creativecommons.org/publicdomain/zero/1.0/) applies to the data made available in this article, unless otherwise stated. 


\section{Background}

Early diagnosis of dengue depends on the detection of the virus by either nucleic acid amplification test (NAAT) [1-4] or detection of dengue virus (DENV) nonstructural protein 1 (NS1) antigen especially that configured into the rapid detection test (RDT) format [5-9]. The NS1 detection method is among the most widely used as it is rapid and simple to perform [9]. The method, however, has its limitation, especially when utilized in dengue endemic regions where secondary dengue is common [5, 9-11]. NS1 assay sensitivity in detection of secondary dengue infection is much lower, hence, may contribute to false negative results $[12,13]$. A complementary detection method is therefore needed [14]. The NAAT has been suggested as the most suitable complementary test since the test allows for direct detection of DENV genome from samples of patients obtained during the viremic phase ( $<5$ days after fever onset). While there are a number of NAATs available, the most common NAAT method for detection of DENV has been the quantitative reverse-transcription polymerase chain reaction (RT-PCR) [15-17]. The test is highly sensitive, specific, and can be easy to perform especially by trained personnel. Unfortunately, due to its requirement for highly specific equipment and reagents, usage of the test has been confined to the well-funded and well-equipped referral laboratories $[18,19]$. The use of NAAT in a resource-limited setting such as peripheral laboratories in many dengue endemic regions of the Southeast Asia is, therefore still limited [20-22]. In recent years, extensive efforts have been undertaken to develop NAAT for the use in these resourcelimited settings for various infectious diseases [23-26]. Implementation of the NAAT as a preferred diagnostic test in this setting, however, remained challenging. The ideal diagnostic test should be a simple, rapid, sensitive, specific, and requires minimal laboratory infrastructure [27]. A more cost-effective NAAT format that met all the aforementioned criteria hence, is needed.

The invention of the isothermal NAAT that requires no or minimum laboratory infrastructure has the potential to overcome the barrier to the use of NAAT in resource-limited setting [28-31]. The isothermal NAAT usually has a simple protocol, easy to perform, does not require a sophisticated instrument, and straightforward result interpretation procedure [32-34]. We recently reported a simple, rapid, sensitive, and specific single tube pan-dengue reverse-transcription recombinase polymerase amplification (RT-RPA) method for early detection of DENV [1]. This method showed comparable sensitivity to the reference real-time RT-PCR test. The dengue RT-RPA assay was performed on an inexpensive portable fluorometer and took only approximately $20 \mathrm{~min}$ to perform with minimal reagent and equipment cost. This NAAT assay hence, has a potential for use in a resourcelimited setting [1]. However, a diagnostic assay with excellent performance in itself is insufficient. The feasibility of a diagnostic test for use in resource-limited setting relies heavily on the robustness of the assay and acceptability by the end users. With this in mind, in the present study, we assessed the operational utilities of the previously described dengue RT-RPA assay, which includes ease to use, the time required to perform the assay, and user acceptability.

\section{Methods \\ Study design}

The usability of performing the RT-RPA assay was assessed among a group of volunteers who were new to the assay. The operational usability of the RT-RPA was evaluated for three focus areas: 1) effectiveness, 2) efficiency and 3) satisfaction. The definitions of the terms of the three areas were as defined below:

1. Effectiveness: the accuracy and completeness with which users can achieve specified goals in a particular environment.

2. Efficiency: the time needed to complete the tasks strictly according to the provided written protocols.

3. Satisfaction: the comfort and acceptability of the work system to the users.

\section{Participants}

Seven researchers were recruited from the Arbovirus Surveillance Laboratory at the Tropical Infectious Disease Research and Education Centre (TIDREC). In addition, we recruited two groups of researchers from i) molecular research laboratories (7 researchers) and ii) antiviral research laboratory (5 researchers) from the Department of Medical Microbiology, Faculty of Medicine, University of Malaya (UM), to participate in the usability testing. A total of 19 researchers with different research experience participated in the study (Additional file 1: Table S1).

\section{Reagent and DENV RNA}

The DENV-specific TwistAmp RT exo lyophilized kit was supplied by TwistDx Ltd., Cambridge, United Kingdom under the European Union FP7 DengueTools agreement 282589 [1]. The lyophilized kit consisting of 1) lyophilized RT-RPA pellet with dengue-specific primers, probes, and fluorescently-tag probes, and 2) customized rehydration buffers consisted of magnesium acetate, potassium acetate, Tris-acetate, and polyethylene glycol 35,000. A random subset of 152 RNA samples extracted from the sera of dengue-suspected patients received between March and May 2015 were used. DENV RNA samples were provided by the WHO Collaborating Centre for Arbovirus Reference \& Research (Dengue/Severe Dengue) (WHO CC) at the UM. The reference RTRPA assay was initially performed by trained laboratory 
personnel of the WHO CC. The WHO CC at UM had previously successfully participated in the WHO WPROconducted EQA program for dengue diagnostics [19]. Results from the reference RT-RPA assay were used as the reference for the current study. Out of the 152 RNA samples, 97 were dengue RT-RPA positive, and 55 were dengue RT-RPA negative samples. In the WHO CC laboratory, all the dengue RT-RPA positive samples showed positive amplification results between 5 and $8 \mathrm{~min}$ after initiating the assay. From these samples, each participant was randomly provided with eight samples to test.

\section{Setting}

The participants were requested to perform the RT-RPA assay following a given standard written protocol (Fig. 1). The participants conducted the assay individually by reading the protocol, and there was no discussion among the participants. During the test, each participant was accompanied by one test facilitator who observed and took notes of the user's conduct of the test. After the run, participants were asked to record the test results. Following completion of the test, participants were requested to complete a posttesting questionnaire adapted from the After-Scenario
Questionnaire developed by Lewis [35] to gauge the user's acceptance and satisfaction towards the test.

\section{Statistical analysis}

Statistical analysis was performed using Prism GraphPad V5.01 [36]. One-way ANOVA was performed to determine the mean difference between the participants with different research experience against the number of deviations from the standard protocol. The same statistical test was also employed to assess the mean difference of groups with 1) different number of deviations from the standard protocol, and 2) different years of research experience against the result accuracy obtained.

\section{Results}

Ease of performing the improved RT-RPA assay

Nineteen participants who had never performed RPA assay were enrolled into this usability testing. The participants were grouped according to their respective laboratories and research experience. Based on the participant's research experience, $31.6 \%(6 / 19)$ of the participants were with less than 1-year of research experience, $36.8 \%(7 / 19)$ with 1 to 5 years of research experience, while the remaining $31.6 \%(6 / 19)$ of the

\section{Dengue RT-RPA Usability Test Protocol}

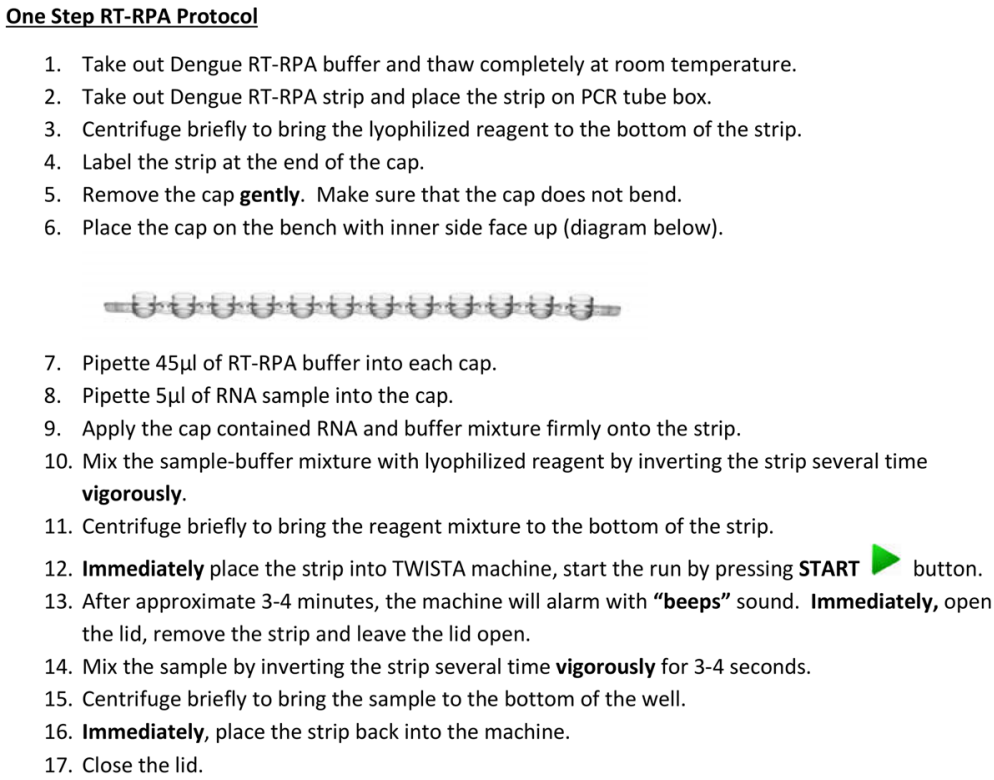

1. Take out Dengue RT-RPA buffer and thaw completely at room temperature.

2. Take out Dengue RT-RPA strip and place the strip on PCR tube box.

3. Centrifuge briefly to bring the lyophilized reagent to the bottom of the strip.

4. Label the strip at the end of the cap.

5. Remove the cap gently. Make sure that the cap does not bend.

6. Place the cap on the bench with inner side face up (diagram below).

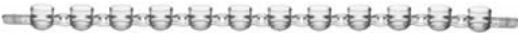

7. Pipette $45 \mu$ l of RT-RPA buffer into each cap.

8. Pipette $5 \mu$ l of RNA sample into the cap.

9. Apply the cap contained RNA and buffer mixture firmly onto the strip.

10. Mix the sample-buffer mixture with lyophilized reagent by inverting the strip several time vigorously.

11. Centrifuge briefly to bring the reagent mixture to the bottom of the strip.

12. Immediately place the strip into TWISTA machine, start the run by pressing START button.

13. After approximate 3-4 minutes, the machine will alarm with "beeps" sound. Immediately, open the lid, remove the strip and leave the lid open.

14. Mix the sample by inverting the strip several time vigorously for 3-4 seconds.

15. Centrifuge briefly to bring the sample to the bottom of the well.

16. Immediately, place the strip back into the machine.

17. Close the lid.

\section{Result Interpretation}

\begin{tabular}{|c|c|}
\hline Target & Interpretation \\
\hline+ & Positive \\
\hline- & Negative \\
\hline$?$ & Failed \\
\hline
\end{tabular}

Fig. 1 Dengue RT-RPA usability test protocol 
Table 1 Number of deviations from the protocol during the test

\begin{tabular}{ll}
\hline Details & $\begin{array}{l}\text { Number (\%) of participant } \\
\text { who performed the test } \\
\text { correctly }\end{array}$ \\
\hline $\begin{array}{l}\text { Number of participants who performed } \\
\text { the test with deviations from protocol }\end{array}$ & \\
a. 0 deviation & $3(15.8 \%)$ \\
b. 1 to 5 deviations & $16(84.2 \%)$ \\
c. More than 5 deviations & $0(0 \%)$ \\
\hline
\end{tabular}

participants had more than 5 years of research experience. All 19 participants completed the test.

\section{Effectiveness}

The effectiveness of the given instruction as the standard protocol to perform the test was evaluated for completeness and accuracy with which the user performed the test (Table 1). During the usability test, of the 19 participants, $15.8 \%$ of the participants performed the test without deviations. The majority of the participants (84.2\%) performed the test with 1 to 5 deviations from the protocol. None of the participants had more than 5 deviations from the protocol during the test.

In order to investigate the possible correlation between the participants' research experience and the deviations that occurred during the test, we analyzed the deviations from the protocol and their respective years of research experience (Table 2). For participants with research experience of less than 1 year, 2 out of 6 participants (33. $3 \%$ ) performed the test without deviations, while $66.7 \%$ of the participants with less than 1-year research experience performed the test with 1 to 5 deviations. None of the participants from the group with less than 1-year research experience performed the test with more than 5 deviations. For the group of participants with 1 to 5 years research experience, all of them (100\%) performed the test with 1 to 5 deviations from the protocol. For participants with more than 5-year research experience, only 1 participant (16.7\%) performed the test without deviations. The remaining of the participants (83. $3 \%)$ performed the assay with 1 to 5 deviations. There were no statistically significant $(p$-value $=0.5545$; oneway ANOVA; Table 3) differences of the mean of deviations occurred during the RT-RPA procedure between the groups of participants with a different year of research experience ( $<1$ year, 1 to 5 years, and $>5$ years).

We analyzed the number of deviations that occurred for each step during the test (Table 4). Results obtained suggested that step 10 and 14 that involved "inverting the strip several times vigorously" showed the highest percentage of deviations from the protocol. 14 out of 19 participants $(73.7 \%)$ performed step 10 with deviations. For step 14, 9 out 19 participants (47.4\%) performed this step with deviations. 3 out of 19 participants (15.8\%) performed step 13 which involved removing the test strip from the machine after the machine alarm gave "beep" sound, with deviations.

Out of 152 samples, we were unable to detect the signal (positive or negative) for two samples (Additional file 2: Table S2). These samples were considered to have failed the RT-RPA assay. These samples were tested by the same participant, suggesting that the failure could be operatordependent. The overall accuracy of the RT-RPA assay was calculated based on the remaining 150 samples. By comparing the test results of the test performed by the participants against the reference test results, the accuracy of test performed by the participants was at $89.3 \%$

Table 2 Number of deviations from protocol during the test for participant with different research experience

\begin{tabular}{ll}
\hline Details & Number (\%) of participant who perform the test correctly \\
\hline pumber of participants with research experience less than 1 year who & \\
a) 0 deviation & $2(33.3 \%)$ \\
b) 1 to 5 deviations & $4(66.7 \%)$ \\
c) More than 5 deviations & $0(0 \%)$ \\
Number of participants with research experience of 1 to 5 years who & \\
performed the test with deviations from the protocol & \\
a) 0 deviation & $0(0 \%)$ \\
b) 1 to 5 deviations & $7(100 \%)$ \\
c) More than 5 deviations & $0(0 \%)$ \\
Number of participant with research experience more than 5 years who & \\
perform the test with deviations from the protocol & \\
a) 0 deviations & $1(16.7 \%)$ \\
b) 1 to 5 deviations & $5(83.3 \%)$ \\
c) More than 5 deviations & $0(0 \%)$ \\
\hline
\end{tabular}


Table 3 Summary of One-way ANOVA analysis

\begin{tabular}{lll}
\hline Type of analysis & $p$-value & Significance $(P<0.05)$ \\
\hline Research experience versus number of deviations & 0.5545 & No \\
Research experience versus result accuracy & 0.1715 & No \\
Number of deviation versus result accuracy & 0.3369 & No \\
\hline
\end{tabular}

(134 /150; Table 5). Of the 16 samples that contributed inconsistent results, seven were false positive, and nine were the false negative. Two out of the false positive samples displayed negative amplification pattern on the readout graphs. These two negative samples somehow were called as 'positive' with the built-in software in Twista ${ }^{\circ}$ fluorometer (TwistDx, UK). While for the false negative samples, eight out of nine showed late amplification pattern, suggesting low target copy number RNA samples.

The One-way ANOVA analysis was also used to investigate the relationship between the number of the deviations from the protocol, and participant's research experience versus the result accuracy. Our results showed that there were no statistically significant mean differences in the result accuracy between groups of participants with different research experience (Table 3). Similarly, the number of deviations from the standard protocol did not significantly affect the result accuracy (Table 3).

\section{Efficiency}

The efficiency of performing the test was evaluated by the time needed for the participants to complete the test

Table 4 Number of deviations of each step during the one-step RT-RPA experiment

\begin{tabular}{lll}
\hline Step & Deviations & \\
\cline { 2 - 3 } & Number & Percentage (\%) \\
\hline 1 & 0 & 0.0 \\
2 & 1 & 5.3 \\
3 & 2 & 10.5 \\
4 & 1 & 5.3 \\
5 & 1 & 5.3 \\
6 & 1 & 5.3 \\
7 & 2 & 10.5 \\
8 & 2 & 10.5 \\
9 & 2 & 10.5 \\
10 & 14 & 73.7 \\
11 & 0 & 0.0 \\
12 & 3 & 15.8 \\
13 & 3 & 15.8 \\
14 & 9 & 47.4 \\
15 & 0 & 0.0 \\
16 & 2 & 10.5 \\
17 & 0 & 0.0 \\
\hline
\end{tabular}

for all eight samples. This included active working time, 15 min amplification time, and time required for interpretation of the results. In the present study, all participants completed the test within 30-45 min.

\section{Satisfaction}

The overall satisfaction of test participants toward the RT-RPA test was assessed by using the post-test questionnaire. The assessment was divided into two parts; the participants were asked to evaluate the satisfaction for i) performing the one-step RT-RPA protocol and ii) interpreting of results. By using 7-point Likert scales, the average score for performing the one-step RT-RPA protocol was 6.21 (Fig. 2; Additional file 3: Table S3). Overall, as high as $90 \%$ of the participants gave positive feedback. Satisfaction for ease of performing the test and time needed to perform were $100 \%$. One participant was not satisfied (rating $=3$ ) with the support documentation supplied with the test.

For interpretation of results, the average score was 6 . 53 , which suggested high positive feedback from the participants (Fig. 3; Additional file 4: Table S4). Satisfaction for the ease to interpret the results and support documentation provided for the test were $100 \%$. One participant gave a score of 4 (moderate) for satisfaction in the time required to interpret the results.

\section{Discussion}

This study represents an analysis of the operational utility of the DENV nucleic acid detection method, RT-RPA in preparation for its possible deployment as a laboratory diagnostic tool. Implementation of a diagnostic test in a new testing environment is highly challenging, particularly in a resource-limited environment. The RTRPA assay was previously described as the most rapid molecular diagnostic tools for detection of DENV [1], but these studies were conducted under laboratory conditions. A well-designed assay, however, does not necessarily define the successfulness of the test adoption in a

Table $\mathbf{5}$ Comparison of the RT-RPA results obtained by the participant against the reference test results

\begin{tabular}{lll}
\hline Reference & \multicolumn{2}{l}{ Participant's RT-RPA test result } \\
\cline { 2 - 3 } & Positive & Negative \\
\hline Positive & 87 & 9 \\
Negative & 7 & 47 \\
\hline
\end{tabular}




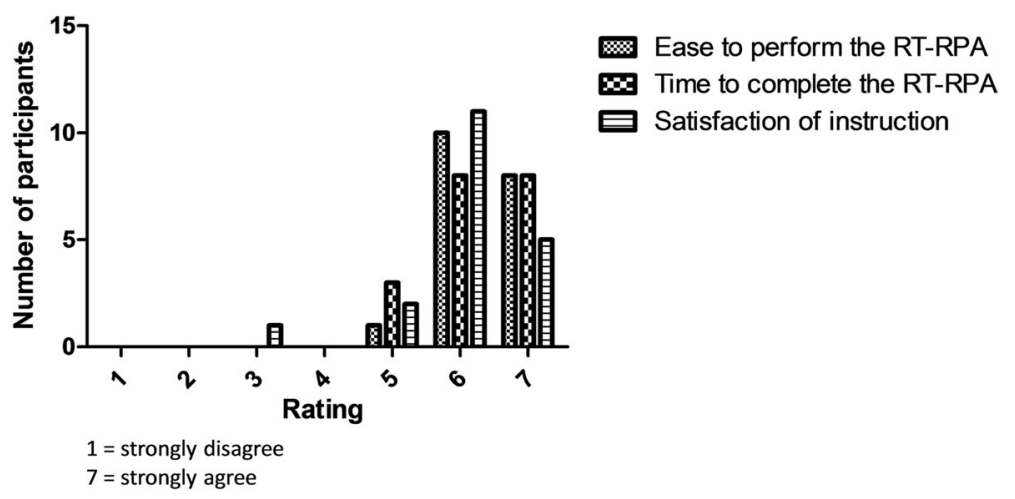

Fig. 2 User satisfaction in performing the one-step RT-RPA. The user satisfaction on one-step RT-RPA assay was evaluated based on three categories; 1) ease to perform the RT-RPA assay, 2) time that needed to complete the RT-RPA assay, and 3) satisfaction of the given written instruction on RT-RPA. The user satisfaction for each category was rated by a scale of 1 to 7 (strongly disagree to strongly agree)

new environment [37]. In the real world context, laboratory setting or field-testing site environment can be highly variable in its infrastructure, availability of instrumentation, and level of experience of the laboratory personnel. The best-developed test may not have a significant impact unless it is well-adapted into the existing diagnostic system and can be properly performed. The challenges are further compounded if it is to be performed in a resource-limited environment where the laboratory infrastructure could be limited. Dengue, in particular, is endemic in many economically developing regions where resources for proper laboratory testing can be scarce outside the major referral healthcare facilities often in the much urbanized capital cities. The operational utility evaluation of the RT-RPA assay prior to its deployment and implementation, hence greatly increase the chances of its successful adoption. The operational utility evaluation would also allow for the necessary adjustments before the assay deployment.
The dengue RT-RPA assay is easy to perform and does not require specialized equipment and high level of skill [38]. In addition to its potential benefit for use in a resource-limited setting, the test could be implemented and adopted into the diagnostic algorithm of a wellestablished laboratory including those at the referral level $[1,39]$. With this in mind, in the study, laboratory personnel with different research experience were recruited from different laboratories. This is important to strengthen the representativeness of the operational utility test. We found no significant differences, however, in the number of deviations from the RT-RPA protocol to the research experience of the participants and their laboratory background in performing the RT-RPA assay. The deviations from the protocol may be random and operator-dependent. In addition to that, we also found that the number of deviations from the protocol showed no significant influences on the result accuracy. The differences in result accuracy were probably linked to the specific technical procedure requiring a mixing step for

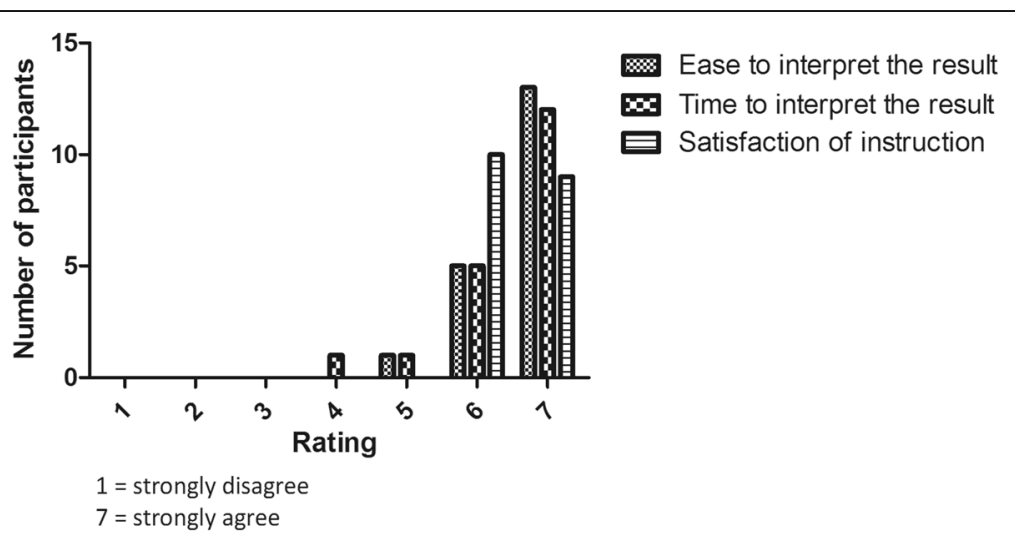

Fig. 3 User satisfaction in the interpretation of results. The user satisfaction on one-step RT-RPA assay was evaluated based on three categories; 1) ease to interpret result, 2) time that needed to interpret result, and 3) satisfaction of the given written instruction on result interpretation. The user satisfaction for each category was rated by a scale of 1 to 7 (strongly disagree to strongly agree) 
the redistribution of the amplicon aggregates formed during the amplification. In the present study, we used the Twista ${ }^{\circ}$ fluorometer (TwistDX, UK) to read the amplification. At about $3 \mathrm{~min}$ and $45 \mathrm{~s}$ after initiation of the test, a beeping alarm would remind the user to remove the tube strip from the fluorometer. Sample mixing was done manually by the operator according to the written instruction [40]. It was at this specific step in the testing process that tendency for deviation was high, especially among new RT-RPA users or in the field setting [41]. This was confirmed in our study, as there were high deviations among users who performed the mixing step, including the way they mixed the sample, the duration of the mixing and the extent of vigor exerted during the mixing, regardless of their research experience. The main reason for these deviations is probably that this specific step is usually not employed in other NAATs. The manual mixing procedure, however, is needed to overcome potential localized depletion of reagents in the area of high RT-RPA activity on the nucleic acid template within the reaction tube [40]. This is a critical step in the amplification cascade to ensure the continuation of the RT-RPA assay and efficient consumption of the reaction mix. In all RT-RPA assays, the mixing step is highly recommended, especially for samples with a low copy number of the target [41]. Obviously, this is the inherent limiting step of the RT-RPA assay needing further attention by the assay developer.

Simplification or elimination of the manual mixing step may help reduce the inter-user variability. One possibility to rid of the manual mixing step is to perform RT-RPA reaction in low volume $(5 \mu \mathrm{l})$ [41]. However, this may not be feasible as at the onset of fever in dengue; there could be as high as $1 \times 10^{8}$ of DENV particles per milliliter of blood [42]. The high concentration of the RNA targets has been shown to affect the RPA assay performance adversely [43]. A recent development of the latest RT-RPA fluorometer, the T-8 isothermal device (TwistDX, UK) that included a built-in magnetic mixing function may obviate the need for the manual mixing [41], and this could help to overcome the current limitation of the assay. With this improvement, the effectiveness of the assay should increase, and the inconsistency due to operator-related factors would be reduced.

User satisfaction is the user's feeling whether the test is easy to perform, the result is easy to interpret, and their readiness to perform the test. The perspective from new users could provide an important comment on the robustness and feasibility of the assay in the actual user application. Generally, user satisfaction is interrelated with the ease of use and efficiency of the assay. An easy to use assay is usually well-accepted by the user. Results from our study suggested very high acceptability of the dengue RT-RPA assay among the new users. This was probably because i) the required reagents were already in lyophilized format, ii) only minimum pipetting steps were needed comparing to the standard RT-PCR or realtime RT-PCR method, and iii) the assay involved only short incubation time of less than 20 min compared to the standard RT-PCR (more than $3 \mathrm{~h}$ ) and real-time RTPCR (45 min to $2 \mathrm{~h}$ ).

\section{Conclusion}

In conclusion, the current study demonstrates the operational utility of the newly developed dengue RT-RPA assay. We showed that the dengue RT-RPA assay was robust, easy to use, efficient, and can easily be performed. Based on our finding, it is likely that the RT-RPA assay will receive high acceptance by most possible new users. Implementation of the dengue RT-RPA could be impactful especially in a resource-limited environment where dengue is endemic, and it could also be a useful complementary test in referral laboratories. Simplification of the RT-RPA protocol by integrating the built-in magnetic mixing function in place of the current manual step should be considered prior to the actual assay deployment.

\section{Additional files}

Additional file 1: Table S1. Number of participants. (DOCX $44 \mathrm{~kb}$ )

Additional file 2: Table S2. Data on RT-RPA amplification. (XLSX $10 \mathrm{~kb}$ ) Additional file 3: Table S3. Data on user satisfaction in performing the one step RT-RPA. (XLSX 9 kb)

Additional file 4: Table S4. Data on user satisfaction in the interpretation of results. (XLSX $9 \mathrm{~kb}$ )

\section{Abbreviations}

DENV: Dengue virus; NAAT: nucleic acid amplification test; NS1: nonstructural protein 1; RDT: Rapid detection test; RT-PCR: reverse-transcription polymerase chain reaction; RT-RPA: reverse-transcription recombinase polymerase amplification; UM: University of Malaya; WHO CC: WHO Collaborating Centre

\section{Funding}

The study was financially supported by the European Union's Seventh Framework Programme (FP7/2007-2013) under the grant agreement 282589-DengueTools.

\section{Availability of data and materials}

All data generated or analyzed in this study are included in this published article and its Additional files.

\section{Authors' contributions}

$S A B$ conceived and designed the study, coordinated the experiments, analyzed and interpreted the data and wrote the manuscript. KKT jointly designed the study with SAB, conducted the experiments, analyzed, interpreted the data, and wrote the manuscript. NSA conducted the experiments, prepared figures and assisted in manuscript writing. CNY, NAA, NIS conducted the experiments and assisted in manuscript writing. BTT and SSS participated in obtaining the reference results for the experiments and assisted in manuscript writing. All authors have read and approved the manuscript for submission. 


\section{Authors' information}

$S A B$ is a senior professor and Director of the Tropical Infectious Diseases Research and Education Centre (TIDREC) at University of Malaya. He is also the director the WHO Collaborating Centre for Arbovirus Reference \& Research (Dengue/Severe Dengue) at the University of Malaya. His research focus is on emerging infectious diseases particularly vector-borne and zoonotic diseases in the tropics. KKT is a Ph.D. candidate in the Department of Medical Microbiology and researcher with the Tropical Infectious Diseases Research and Education Centre (TIDREC) at University of Malaya. Her research interest is in infectious diseases.

\section{Ethics approval and consent to participate}

The study was approved by the Medical Ethics Committee of the University Malaya Medical Centre (UMMC) with MEC Ref No of 908.11. Written consents have been obtained from all participants who took part in the study.

\section{Consent for publication}

All authors have agreed to submissionof this manuscript.

\section{Competing interests}

The authors declare that they have no competing interests.

\section{Publisher's Note}

Springer Nature remains neutral with regard to jurisdictional claims in published maps and institutional affiliations.

\section{Author details}

${ }^{1}$ Tropical Infectious Diseases Research and Education Centre (TIDREC), University of Malaya, 50603 Kuala Lumpur, Malaysia. ${ }^{2}$ WHO Collaborating Centre for Arbovirus Research and Reference (Dengue and Severe Dengue), University of Malaya, 50603 Kuala Lumpur, Malaysia. ${ }^{3}$ Department of Medical Microbiology, Faculty of Medicine, University of Malaya, 50603 Kuala Lumpur, Malaysia.

Received: 11 July 2017 Accepted: 26 March 2018

Published online: 11 April 2018

\section{References}

1. Teoh BT, Sam SS, Tan KK, Danlami MB, Shu MH, Johari J, Hooi PS, Brooks D, Piepenburg O, Nentwich O, et al. Early detection of dengue virus by use of reverse transcription-recombinase polymerase amplification. J Clin Microbiol. 2015;53(3):830-7.

2. Lau YL, Lai MY, Teoh BT, Abd-Jamil J, Johari J, Sam SS, Tan KK, AbuBakar S. Colorimetric detection of dengue by single tube reverse-transcription-loopmediated isothermal amplification. PLoS One. 2015;10(9):e0138694.

3. Teoh BT, Sam SS, Tan KK, Johari J, Danlami MB, Hooi PS, Md-Esa R, Abubakar S. Detection of dengue viruses using reverse transcription-loop-mediated isothermal amplification. BMC Infect Dis. 2013;13(1):387.

4. Tan KK, Johari J, Abd-Jamil J, Zulkifle NI, Sulaiman S, Zainal N, Ab Rahman HA, Sam SS, Teoh BT, AbuBakar S. Comparison of real time reverse transcription-polymerase chain reaction assays for the detection of dengue virus. JUMMEC. 2013;16:16.

5. Teoh BT, Sam SS, Tan KK, Johari J, Abd-Jamil J, AbuBakar S. NS1 rapid test for detection of dengue virus infection. Trop Med Int Health. 2015;20(S1): 114.

6. Pal S, Dauner AL, Mitra I, Forshey BM, Garcia P, Morrison AC, Halsey ES, Kochel TJ, WU SJ. Evaluation of dengue NS1 antigen rapid tests and ELISA kits using clinical samples. PLoS One. 2014;9(11):e113411.

7. Kassim FM, Izati MN, TgRogayah T, Apandi YM, Saat Z. Use of dengue NS1 antigen for early diagnosis of dengue virus infection. Southeast Asian J Trop Med Public Health. 2011:42(3):562-9.

8. Blacksell SD, Jarman RG, Bailey MS, Tanganuchitcharnchai A, Jenjaroen K, Gibbons RV, Paris DH, Premaratna R, de Silva HJ, Lalloo DG, et al. Evaluation of six commercial point-of-care tests for diagnosis of acute dengue infections: the need for combining NS1 antigen and IgM/lgG antibody detection to achieve acceptable levels of accuracy. Clin Vaccine Immunol. 2011;18(12):2095-101.

9. Tricou V, Vu HT, Quynh NV, Nguyen CV, Tran HT, Farrar J, Wills B, Simmons CP. Comparison of two dengue NS1 rapid tests for sensitivity, specificity and relationship to viraemia and antibody responses. BMC Infect Dis. 2010;10:142.

10. Hunsperger EA, Yoksan S, Buchy P, Nguyen VC, Sekaran SD, Enria DA, Vazquez S, Cartozian E, Pelegrino JL, Artsob H, et al. Evaluation of commercially available diagnostic tests for the detection of dengue virus NS1 antigen and anti-dengue virus IgM antibody. PLoS Negl Trop Dis. 2014; 8(10):e3171

11. Felix $A C$, Romano CM, Centrone Cde C, Rodrigues CL, Villas-Boas L, Araujo ES, de Matos AM, Carvalho Kl, Martelli CM, Kallas EG, et al. Low sensitivity of NS1 protein tests evidenced during a dengue type 2 virus outbreak in Santos, Brazil, in 2010. Clin Vaccine Immunol. 2012;19(12):1972-6.

12. Hermann $\mathrm{LL}$, Thaisomboonsuk $B$, Poolpanichupatam $\mathrm{Y}$, Jarman RG, Kalayanarooj S, Nisalak A, Yoon IK, Fernandez S. Evaluation of a dengue NSI antigen detection assay sensitivity and specificity for the diagnosis of acute dengue virus infection. PLoS Negl Trop Dis. 2014;8(10):e3193.

13. Osorio L, Ramirez M, Bonelo A, Villar LA, Parra B. Comparison of the diagnostic accuracy of commercial NS1-based diagnostic tests for early dengue infection. Virology J. 2010;7:361.

14. Teoh BT, Sam SS, Tan KK, Johari J, Abd-Jamil J, Hooi PS, AbuBakar S. The use of NS1 rapid diagnostic test and GRT-PCR to complement IgM ELISA for improved dengue diagnosis from single specimen. Sci Rep. 2016;6:27663.

15. Santiago GA, Vergne E, Quiles Y, Cosme J, Vazquez J, Medina JF, Medina F, Colon C, Margolis H, Munoz-Jordan JL. Analytical and clinical performance of the CDC real time RT-PCR assay for detection and typing of dengue virus. PLoS Negl Trop Dis. 2013;7(7):e2311.

16. Waggoner JJ, Abeynayake J, Sahoo MK, Gresh L, Tellez Y, Gonzalez K, Ballesteros G, Guo FP, Balmaseda A, Karunaratne K, et al. Comparison of the FDA-approved CDC DENV-1-4 real-time reverse transcription-PCR with a laboratory-developed assay for dengue virus detection and serotyping. J Clin Microbiol. 2013;51(10):3418-20.

17. Najioullah F, Viron F, Cesaire R. Evaluation of four commercial real-time RTPCR kits for the detection of dengue viruses in clinical samples. Virol J. 2014; 11:164.

18. Domingo C, Niedrig M, Teichmann A, Kaiser M, Rumer L, Jarman RG, Donoso-Mantke $\mathrm{O}$. 2nd international external quality control assessment for the molecular diagnosis of dengue infections. PLoS Negl Trop Dis. 2010; 4(10):e833

19. Pok KY, Squires RC, Tan LK, Takasaki T, Abubakar S, Hasebe F, Partridge Lee CK, Lo J, Aaskov J, et al. First round of external quality assessment of dengue diagnostics in the WHO western Pacific region, 2013. Western Pac Surveill Response J. 2015;6(2):73-81.

20. Mabey D, Peeling RW, Ustianowski A, Perkins MD. Diagnostics for the developing world. Nat Rev Microbiol. 2004;2(3):231-40.

21. Niemz A, Ferguson TM, Boyle DS. Point-of-care nucleic acid testing for infectious diseases. Trends Biotechnol. 2011;29(5):240-50.

22. Drain PK, Hyle EP, Noubary F, Freedberg KA, Wilson D, Bishai WR, Rodriguez W, Bassett IV. Diagnostic point-of-care tests in resource-limited settings. Lancet Infect Dis. 2014;14(3):239-49.

23. Boyle DS, McNerney R, Teng Low H, Leader BT, Perez-Osorio AC, Meyer JC, O'Sullivan DM, Brooks DG, Piepenburg O, Forrest MS. Rapid detection of Mycobacterium tuberculosis by recombinase polymerase amplification. PLoS One. 2014;9(8):e103091.

24. Euler $\mathrm{M}$, Wang $\mathrm{Y}$, Nentwich $\mathrm{O}$, Piepenburg $\mathrm{O}$, Hufert FT, Weidmann $\mathrm{M}$. Recombinase polymerase amplification assay for rapid detection of Rift Valley fever virus. J Clin virol. 2012;54(4):308-12.

25. Crannell Z, Castellanos-Gonzalez A, Nair G, Mejia R, White AC, RichardsKortum R. Multiplexed recombinase polymerase amplification assay to detect intestinal protozoa. Anal Chem. 2016;88(3):1610-6.

26. Kersting S, Rausch V, Bier FF, von Nickisch-Rosenegk M. Rapid detection of plasmodium falciparum with isothermal recombinase polymerase amplification and lateral flow analysis. Malar J. 2014;13:99.

27. Pai NP, Vadnais C, Denkinger C, Engel N, Pai M. Point-of-care testing for infectious diseases: diversity, complexity, and barriers in low- and middleincome countries. PLoS Med. 2012;9(9):e1001306.

28. Liljander A, Yu M, O'Brien E, Heller M, Nepper JF, Weibel DB, Gluecks I, Younan M, Frey J, Falquet L, et al. Field-applicable recombinase polymerase amplification assay for rapid detection of mycoplasma capricolum subsp. capripneumoniae. J Clin Microbiol. 2015;53(9):2810-5.

29. Oriero EC, Jacobs J, Van Geertruyden JP, Nwakanma D, D'Alessandro U. Molecular-based isothermal tests for field diagnosis of malaria and their potential contribution to malaria elimination. J Antimicrob Chemother. 2015;70(1):2-13.

30. Mondal D, Ghosh P, Khan MA, Hossain F, Bohlken-Fascher S, Matlashewski G, Kroeger A, Olliaro P. Abd El Wahed a: mobile suitcase laboratory for rapid detection of Leishmania donovani using recombinase polymerase amplification assay. Parasit Vectors. 2016;9(1):281. 
31. Lillis L, Lehman D, Singhal MC, Cantera J, Singleton J, Labarre P, Toyama A, Piepenburg O, Parker M, Wood R, et al. Non-instrumented incubation of a recombinase polymerase amplification assay for the rapid and sensitive detection of proviral HIV-1 DNA. PLoS One. 2014;9(9):e108189.

32. Boehme CC, Nabeta P, Henostroza G, Raqib R, Rahim Z, Gerhardt M, Sanga E, Hoelscher M, Notomi T, Hase T, et al. Operational feasibility of using loopmediated isothermal amplification for diagnosis of pulmonary tuberculosis in microscopy centers of developing countries. J Clin Microbiol. 2007;45(6): 1936-40.

33. Spinelli O, Rambaldi A, Rigo F, Zanghi P, D'Agostini E, Amicarelli G, Colotta F, Divona M, Ciardi C, Coco FL, et al. Simple, rapid and accurate molecular diagnosis of acute promyelocytic leukemia by loop mediated amplification technology. Oncoscience. 2015;2(1):50-8.

34. Daher RK, Stewart G, Boissinot M, Bergeron MG. Recombinase polymerase amplification for diagnostic applications. Clin Chem. 2016;62(7):947-58.

35. Lewis JR. IBM computer usability satisfaction questionnaires: psychometric evaluation and instructions for use. Int J Hum Comput Interact. 1995;7(1): 57-78.

36. Prism G. Version 5.01. San Diego, CA, USA: GraphPad Software Inc; 2007.

37. Schiff GD, Kim S, Abrams R, Cosby K, Lambert B, Elstein AS, Hasler S, Krosnjar $\mathrm{N}$, Odwazny R, Wisniewski MF et al: Diagnosing diagnosis errors: lessons from a multi-institutional collaborative project. In: Advances in Patient Safety: From Research to Implementation (Volume 2: Concepts and Methodology). Edn. Edited by Henriksen K, Battles JB, Marks ES, Lewin DI. Rockville (MD); 2005.

38. Crannell ZA, Rohrman B, Richards-Kortum R. Equipment-free incubation of recombinase polymerase amplification reactions using body heat. PLoS One. 2014;9(11):e112146.

39. Abd El Wahed A, Patel P, Faye O, Thaloengsok S, Heidenreich D, Matangkasombut P, Manopwisedjaroen K, Sakuntabhai A, Sall AA, Hufert FT, et al. Recombinase polymerase amplification assay for rapid diagnostics of dengue infection. PLoS One. 2015;10(6):e0129682.

40. TwistDx. TwistAmp combined instruction manual. Pamphlet: Ref Type; 2014.

41. Lillis L, Siverson J, Lee A, Cantera J, Parker M, Piepenburg O, Lehman DA, Boyle DS. Factors influencing recombinase polymerase amplification (RPA) assay outcomes at point of care. Mol Cell Probes. 2016;30(2):74-8.

42. Vaughn DW, Green S, Kalayanarooj S, Innis BL, Nimmannitya S, Suntayakorn S, Endy TP, Raengsakulrach B, Rothman AL, Ennis FA, et al. Dengue viremia titer, antibody response pattern, and virus serotype correlate with disease severity. J Infect Dis. 2000;181(1):2-9.

43. Rohrman B, Richards-Kortum R. Inhibition of recombinase polymerase amplification by background DNA: a lateral flow-based method for enriching target DNA. Anal Chem. 2015;87(3):1963-7.

\section{Submit your next manuscript to BioMed Central and we will help you at every step:}

- We accept pre-submission inquiries

- Our selector tool helps you to find the most relevant journal

- We provide round the clock customer support

- Convenient online submission

- Thorough peer review

- Inclusion in PubMed and all major indexing services

- Maximum visibility for your research

Submit your manuscript at www.biomedcentral.com/submit

) Biomed Central 